\title{
Electron Transport Properties of Single-Molecule-Bearing Multiple Redox Levels Studied by EC-STM/STS
}

\author{
Paolo Petrangolini, ${ }^{+, \neq}$Andrea Alessandrini, ${ }^{+, \neq}$Maria Luisa Navacchia, ${ }^{\S}$ Massimo Luigi Capobianco, ${ }^{\S}$ and \\ Paolo Facci, ${ }^{*,+}$ \\ ${ }^{+}$Centro S3, CNR-Istituto di Nanoscienze, Via Campi 213/A, 41125 Modena, Italy \\ ${ }^{\ddagger}$ Department of Physics, University of Modena and Reggio Emilia, Via Campi 213/A, 41125 Modena, Italy \\ ${ }^{\S}$ CNR-Istituto per la Sintesi Organica e la Fotoreattività (ISOF), Via P. Gobetti 101, 40129 Bologna, Italy
}

Supporting Information

ABSTRACT: Multielectron systems as possible components of molecular electronics devices are attracting compelling experimental and theoretical interest. Here we studied by electrochemical scanning tunneling techniques (EC-STMicroscopy and EC-STSpectroscopy) the electron transport properties of a redox molecule endowed with two redox levels, namely, the hydroquinone/quinone $\left(\mathrm{H}_{2} \mathrm{Q} / \mathrm{Q}\right)$ couple. By forming self-assembled monolayers on $\mathrm{Au}(111)$ of oligo-phenylene-vinylene (OPV) derivatized $\mathrm{H}_{2} \mathrm{Q} / \mathrm{Q}$ moieties, we were able to explore the features of the tunneling current/overpotential relation in the EC-STS setup. The behavior of the tunneling current sheds light onto the mechanism of electron
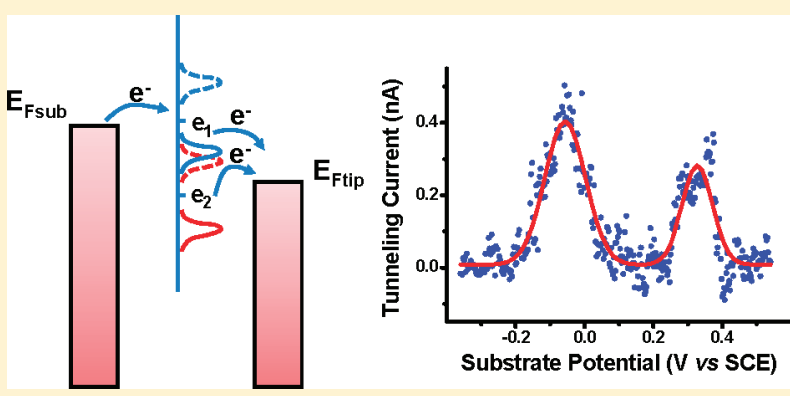

transport involving the redox levels of the $\mathrm{H}_{2} \mathrm{Q} / \mathrm{Q}$ redox pair coupled to tip and substrate electrodes.

\section{INTRODUCTION}

The exploitation of single molecules or single monomolecular layers as active elements of electronic circuits is the main goal of molecular electronics. ${ }^{1,2}$ Single molecules can act as switches or rectifiers or can show features of negative differential conductance. ${ }^{3,4}$ Most importantly, their properties could be specifically designed, by synthetic chemistry, to meet the desired requirements. ${ }^{5}$ Current main efforts in molecular electronics are directed to understanding electronic transport mechanisms, including issues related to the interface between metallic or semiconductor electrodes and molecules and the role of molecular electronic states in electron transfer mechanisms. ${ }^{6}$ Among the different types of molecules that have been tested for their potentiality in molecular electronics devices, redox-active molecular species appear to be very promising. ${ }^{7}$ Many theoretical and experimental efforts have been devoted to studying the electron transport process in redox active molecules connecting two metal electrodes in the electrochemical scanning tunneling microscope setup. ${ }^{8-15}$ The scanning tunneling microscope offers the possibility of combining imaging with spectroscopic analysis of single molecules. The presence of an electrolytic solution allows us to perform a gating of the molecular conductivity without the need of positioning a third nanoelectrode near to source and drain electrodes (i.e., tip and substrate). ${ }^{16}$ Indeed, in many cases, the electrolyte gating effect has been demonstrated. This effect consists of a tunneling current enhancement at fixed bias voltage obtained when the overpotential is in the proximity of the equilibrium potential of the redox group. In a recent work, we have studied the current/overpotential characteristics for the hydroquinone/quinone redox couple tethered to $\mathrm{Au}(111)$ substrate in an electrochemical scanning tunneling microscopy (EC-STM) configuration. ${ }^{17}$ The peculiarity of this redox couple is that its oxidation/reduction reaction involves globally the exchange of two electrons and two protons. The system is interesting in light of the relevance of multielectron systems as components for molecular electronics devices. However, the global electron transfer mechanism, as measured in an ECSTM setup, remained somehow elusive. Dealing with redoxmolecules, it is a ready-reference to compare the results of single molecule conductance with those performed with standard electrochemical techniques that deal with electron transfer rates. ${ }^{18}$ In fact, some important parameters for the interpretation of singlemolecule conductivity experiments on redox-active components can be suggested by cyclic voltammogram analysis. The relation between the two types of measurements is currently under discussion. ${ }^{18}$ In the specific case of the hydroquinone/benzoquinone redox couple, a cyclic voltammetry analysis of a self-assembled monolayer (SAM) of derivatized hydroquinone moieties in buffered solution in a $\mathrm{pH}$ range from 4 to 9 shows the presence of a single reduction/oxidation wave involving the exchange of two electrons. ${ }^{19}$ When the same molecular construct is analyzed by EC-STM as a function of the overpotential, two regions of tunneling current enhancement are found. ${ }^{17}$ This different behavior

\footnotetext{
Received: August 29, 2011

Revised: September 2, 2011

Published: September 02, 2011
} 
Chart 1. Structure of 4-(2',5'-Dihydroxystyryl)benzyl Thioacetate (1)

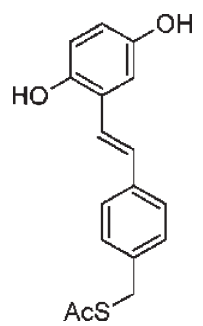

points to a different electron transfer mechanism in the two experimental setups. In the specific case of the hydroquinone/ benzoquinone redox couple, protons are also involved in the reaction. This feature makes the oxidation/reduction reactions highly dependent on the environment, complicating even more the mechanistic description of the process.

In ref 17 , we interpreted the appearance of two regions of tunneling enhancement as a function of the substrate overpotential as mainly due to the effect of two processes of electron transfer, each one involving a single electron, according to the nine-membered scheme of hydroquinone/benzoquinone reaction developed by Laviron. ${ }^{20,21}$ The two peaks observed in the tunneling current/overpotential spectroscopy were interpreted as being due to the presence of the two redox equilibria, even if a possible role of a coulomb blockade mechanism in the transfer mechanism was also conjectured. In the present work, we have changed the alkyl linker between the substrate and the quinone moiety for an oligo-phenylene-vinylene one to modify the electronic coupling and the electron transfer rate between the hydroquinone and the substrate, evaluating the effects of this modification both by cyclic voltammetry and EC-STM/STS (scanning tunneling spectroscopy). The choice of the new molecule is mainly suggested by the possibility of exploring the role of the conjectured coulomb blockade mechanism by modifying the coupling of the redox moiety with the substrate. The new molecule should show a decreased separation between the two current enhancement regions with respect to the previous molecule. This condition should allow us to test the coulomb blockade hypothesis and, at the same time, some predictions of theories developed for the interfacial electron transfer mechanism involving multiple redox levels. ${ }^{22,23}$ In particular, a small separation between the peaks would permit us to study the features of the tunneling current/ overpotential relation for a bias voltage larger than peak separation. This investigation would shed further light on the electron transfer mechanism involved in the system at issue.

\section{EXPERIMENTAL SECTION}

2.1. Sample Preparation. 4-(2',5'-Dihydroxystyryl)benzyl thioacetate (Chart 1 , compound $\mathbf{1}$ ) was synthesized according to the procedure by Trammell et al. ${ }^{24} \mathrm{Au}(111)$ substrates were prepared by evaporating a $150 \mathrm{~nm}$ thick gold layer on a high vacuum prebaked $\left(450^{\circ} \mathrm{C}\right)$ mica substrate and subsequently annealing them at $450^{\circ} \mathrm{C}$ for $4 \mathrm{~h}$ in high vacuum. For assembling the hydroquinone adlayers, a gold substrate was flame-annealed and immediately immersed in an ethanol solution containing the specific molecules to form the SAM. For EC-STM imaging, an SAM was assembled on $\mathrm{Au}(111)$ starting from an ethanol solution composed of $10^{-5} \mathrm{M}$ 6-mercapto-1-hexanol $(\mathrm{MCH})$ following overnight incubation. After incubation, the sample was rinsed in abundant ethanol and immersed in a $10^{-5} \mathrm{M} 4-\left(2^{\prime}, 5^{\prime}\right.$-dihydroxystyryl)benzyl thioacetate ethanol solution for $60 \mathrm{~min} .^{25}$ The sample was then rinsed in ethanol and mounted in the EC-STM cell. The imaging buffer was $50 \mathrm{mM} \mathrm{NH}_{4} \mathrm{Ac}, \mathrm{pH} 4.6$ or 7.6. This procedure allowed us to obtain an adlayer that showed, in the EC-STM images, the presence of bumps protruding from a background. The bumps can be interpreted as single molecules or small islands of derivatized hydroquinone molecules protruding above the MCH layer. For EC-STS, we assembled samples by incubating freshly annealed $\mathrm{Au}(111)$ substrates in a $10^{-5} \mathrm{M}$ 4-(2',5'-dihydroxystyryl)benzyl thioacetate ethanol solution overnight. Then, the sample was abundantly rinsed in ethanol and installed in the EC-STM cell. The buffers used for EC-STS were $50 \mathrm{mM} \mathrm{NH}_{4} \mathrm{Ac}, \mathrm{pH} 4.6$ and 7.6.

2.2. Electrochemical Characterization, EC-STM Imaging and Scanning Tunneling Spectroscopy. The derivatized hydroquinone SAMs were characterized by cyclic voltammetry. Electrochemical data were acquired under the same conditions as those for EC-STM and STS to be best compared. The buffers used for cyclic voltammetry were $50 \mathrm{mM} \mathrm{NH}_{4} \mathrm{Ac}$ at two different $\mathrm{pH}$ values: 4.6 and 7.6.

EC-STM imaging was performed by a PicoSPM microscope (Molecular Imaging) equipped with a bipotentiostat. The bipotentiostat allowed us to control independently the potential of substrate and tip with respect to a quasi-reference silver wire. The tip was prepared by electrochemically etching a $\mathrm{Pt} / \mathrm{Ir}(80 / 20)$ wire, and it was coated with Apiezon wax to reduce leakage currents to values smaller than 5-10 pA. As a counter electrode, a Pt wire was used. The potential of the silver wire was checked against an SCE reference electrode at the beginning and at the end of each experimental session, and all of the data in the following are referred to SCE. For spectroscopy-like imaging, a sequence of images of the same sample region was acquired at constant bias for different values of substrate potential. The apparent height variation of the hydroquinone-terminated molecules upon varying the substrate potential was measured against the electrochemically inactive MCH molecules. For EC-STS, the variation of the tunneling current upon substrate potential $\left(V_{s}\right)$ was measured at fixed height and lateral tip position on the sample. To this aim, tip-substrate approach was performed as usual at a given tunneling current set point, and the feedback loop was then switched off. During approach, a substrate potential value was set according to spectroscopy-like imaging information to avoid tunneling current enhancement. The substrate potential was then swept alternatively toward positive and negative direction at scan rate of $0.05 \mathrm{~V} / \mathrm{s}$ to cross the redox potential of the hydroquinone/benzoquinone couple. The comparison of the tunneling current at the beginning and at the end of the sweep was used as a probe of vertical stability of the tip/substrate position. STS experiments were also performed at two different bias voltages: 100 and $200 \mathrm{mV}$.

\section{RESULTS AND DISCUSSION}

In ref 17 , we measured the electrochemical scanning tunneling spectroscopy signal on 2-(6-mercaptoalkyl)hydroquinone molecules on $\mathrm{Au}(111)$ for two different $\mathrm{pH}$ values: 4.6 and 7.6. The STS analysis was performed on a SAM of that molecule, and for both $\mathrm{pH}$ values, the presence of two regions of tunneling current enhancement as a function of substrate potential was observed. By changing $\mathrm{pH}$, the voltage position of the tunneling current 


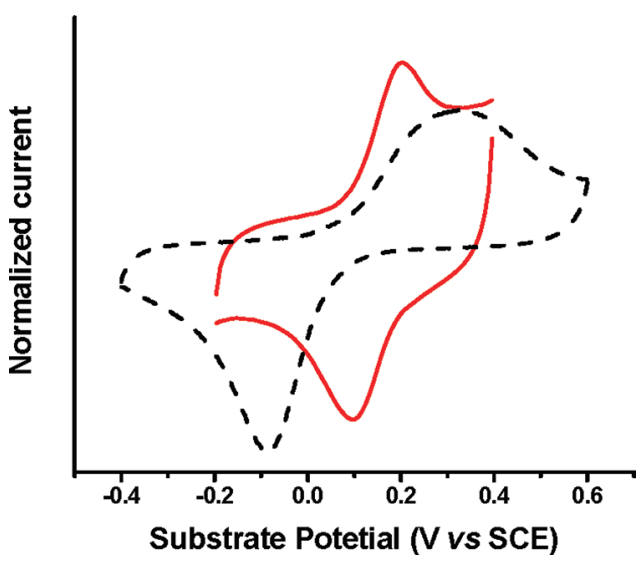

Figure 1. Cyclic voltammograms of 2-(6-mercaptoalkyl)hydroquinone SAMs on gold (dashed line) and 4-(2',5'-dihydroxystyryl)benzyl thioacetate (continuous line). The voltammograms have been obtained in $50 \mathrm{mM} \mathrm{NH}_{4} \mathrm{Ac}, \mathrm{pH} 4.6$ at a scan rate of $0.5 \mathrm{~V} / \mathrm{s}$.

maxima changed: they were nearer to each other for higher $\mathrm{pH}$. A possible role of a coulomb blockade mechanism was suggested as an explanation for the appearance of two regions of tunneling current enhancement. Many theoretical investigations dealing with the presence of two redox levels in the gap between the tip and the substrate in EC-STM prompted that an information-rich experiment is that in which the bias voltage is changed relatively to the energy separation between the two levels. ${ }^{22,23,26,27}$ Inspired by these ideas, we chose to work with 4-( $2^{\prime}, 5^{\prime}$-dihydroxystyryl)benzyl thioacetate; given its delocalized bridge, it should ensure a smaller separation of the two maxima if a coulomb blockade mechanism is present in the observed phenomenon and because of its possibly increased electronic coupling and higher electron transfer rate to the substrate. In any case, the opportunity of having the two peaks nearer to each other would enable studying features connected with an applied bias voltage higher than the potential separation between the two peaks.

3.1. Electrochemical Characterization of 4-(2',5'-Dihydroxystyryl)benzyl Thioacetate SAM on Gold. We first characterized SAMs of the new molecule on gold by cyclic voltammetry. Figure 1 shows a cyclic voltammogram of the molecule (1) compared with that of the molecule used in ref 17 . The two molecules show different electron transfer rates due to the different linker with the substrate. Further electrochemical characterizations, including dependence of the peak current and position on the scan rate, and dependence on $\mathrm{pH}$ are reported in the Supporting Information file. From these data, it results that both the anodic and cathodic peaks involve an average exchange of 1.5 electrons, and the redox potential shifts by $-0.059 \mathrm{~V} / \mathrm{pH}$, in agreement with a Nernstian redox species involving the exchange of two protons and two electrons.

3.2. EC-STM and EC-STS Investigation of 4-( $2^{\prime}, 5^{\prime}$-Dihydroxystyryl)benzyl Thioacetate on Gold. We then passed to the characterization of the complex (1) by both spectroscopylike imaging and scanning tunneling spectroscopy in electrochemical environment. A mixed SAM composed of hydroquinone with the OPV linker and MCH was assembled on a $\mathrm{Au}(111)$ substrate for spectroscopy-like imaging. Figure 2 reports ECSTM images of the molecular construct. The mixed monolayer was assembled by the substitution technique starting from an MCH SAM. ${ }^{25}$ Figure 2a shows the MCH layer with the presence of pits, one-atom-layer deep, typical of thiolated SAMs on gold.
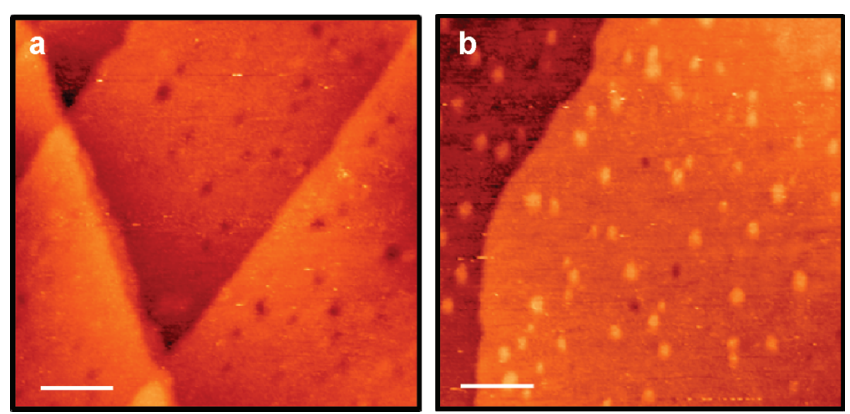

Figure 2. Molecular construct for EC-STM spectroscopy-like imaging. (a) SAM of 6-mercapto-1-hexanol (MCH) is assembled on an $\mathrm{Au}(111)$ surface. Scale bar: $20 \mathrm{~nm}$. (b) By exploiting the substitution technique, 4 - $\left(2^{\prime}, 5^{\prime}\right.$-dihydroxystyryl $)$ benzyl thioacetate molecules are included in the SAM. The visible protruding bumps can be associated with single molecules or small islands of 4-(2',5'-dihydroxystyryl)benzyl thioacetate. Scale bar: $30 \mathrm{~nm}$. Both images have been acquired in $50 \mathrm{mM}$ $\mathrm{NH}_{4} \mathrm{Ac}$, bias $400 \mathrm{mV}$, substrate potential: $-60 \mathrm{mV}$ versus SCE.

After an incubation of the preformed $\mathrm{MCH}$ layer with complex (1), we observed the presence of both pits and molecules protruding from the $\mathrm{MCH}$ layer, which we attributed to derivatized hydroquinone molecules (Figure 2b). (Their density in the ECSTM images is proportional to the incubation time and hydroquinone concentration in the incubation solution.)

Performing a sequence of images at constant bias $(400 \mathrm{mV}$, tip positive) and varying substrate potential with respect to the reference electrode, we obtained the images reported in Figure 3. This sequence, spanning a substrate potential range from -300 to $+500 \mathrm{mV}$ versus SCE, clearly shows that the apparent height of the spots with respect to the $\mathrm{MCH}$ layer depends on substrate potential (Figure $3 a-f$ ). A statistical analysis of the apparent height based on the measurements of 35 molecules showed the presence of two regions of apparent height increase (Figure 3g). Moreover, considering the apparent height in regions of no enhancement, it turned out that an overall increase took place if one goes from the reduced to the oxidized molecular state according to previous results on a similar molecule. ${ }^{28}$

Comparing the above results with those obtained in our previous work on 2-(6-mercaptoalkyl)hydroquinone, we note that two regions of tunneling current enhancement are again visible, but now the peak separation is $400 \mathrm{mV}$ at variance with the previous feature of $600 \mathrm{mV}$ under similar conditions. This result highlights that the delocalized bridge of the hydroquinone moiety moves the two peaks closer to each other. We then passed to EC-STS on a SAM composed only of the OPV-derivatized quinone molecule to get direct access to the tunneling current. By this technique, one can obtain the tunneling current/overpotential characteristic curve that is the typical output of theories analyzing the behavior of redox molecules in an EC-STM setup. ${ }^{29-31}$ The results are reported in Figure 4. Similarly to what we observed in the case of spectroscopy-like imaging on complex (1) of this work and on 2-(6-mercaptoalkyl)hydroquinone, ${ }^{17}$ we found two regions of tunneling current enhancement. The two regions were positioned at approximately the same potential $(-50$ and $+350 \mathrm{mV}$ vs SCE) as that in EC-STM. A slight rigid shift toward higher substrate potentials was observed for the peaks in the case of spectroscopy-like imaging. This behavior could be related to the different bias voltage used in the two experiments. ${ }^{17}$ Moreover, given the strong dependence of redox potential on $\mathrm{pH}$ for the hydroquinone/benzoquinone redox couple, we studied the behavior 

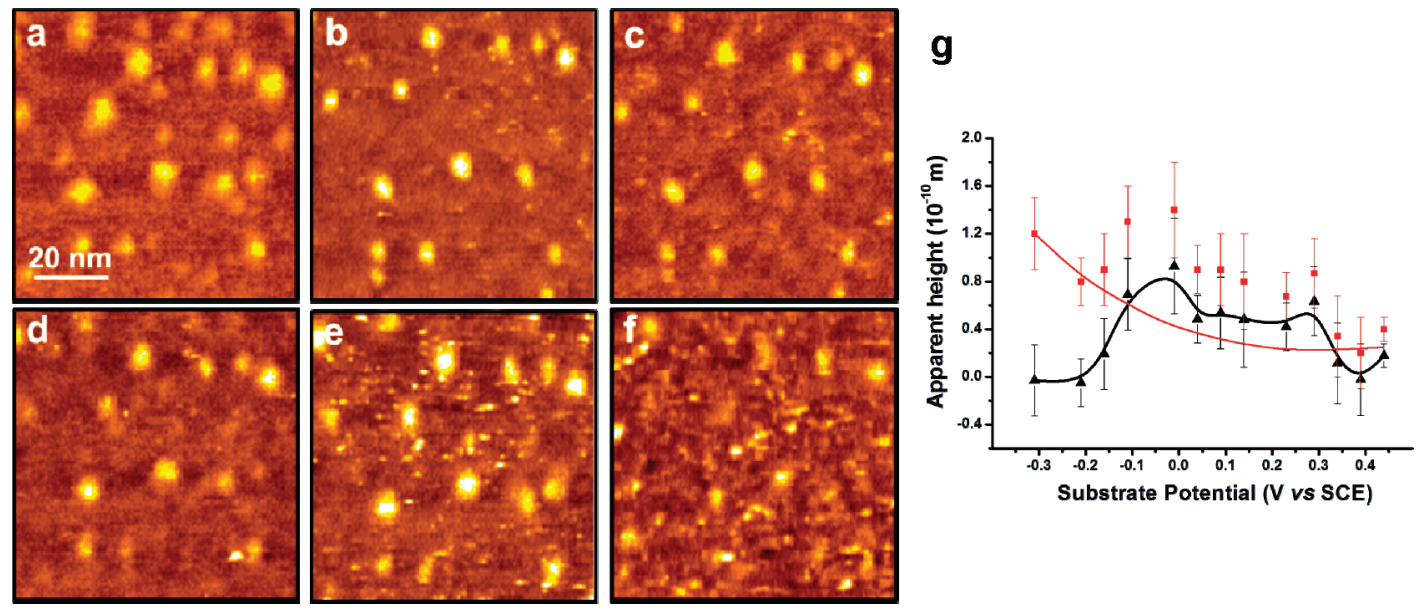

Figure 3. Spectroscopy-like imaging of a mixed SAM of $\mathrm{MCH}$ and 1. From a-f, the images have been acquired at the following substrate potentials: (a) -310 , (b) -60, (c) +40 , (d) 90, (e) +290 , and (f) $490 \mathrm{mV}$ all versus SCE. Buffer: $50 \mathrm{mM} \mathrm{NH}_{4}$ Ac, bias $400 \mathrm{mV}$. (g) Apparent height of the bumps with respect to the $\mathrm{MCH}$ layer from a population of 35 spots: Red squares are the measured apparent heights from EC-STM images. The continuous red line represents the background increase in the apparent height that occurs when the oxidation state of the molecule changes. Black triangles are obtained from the red ones after background subtraction. The overlaid continuous line is a guide for the eye.
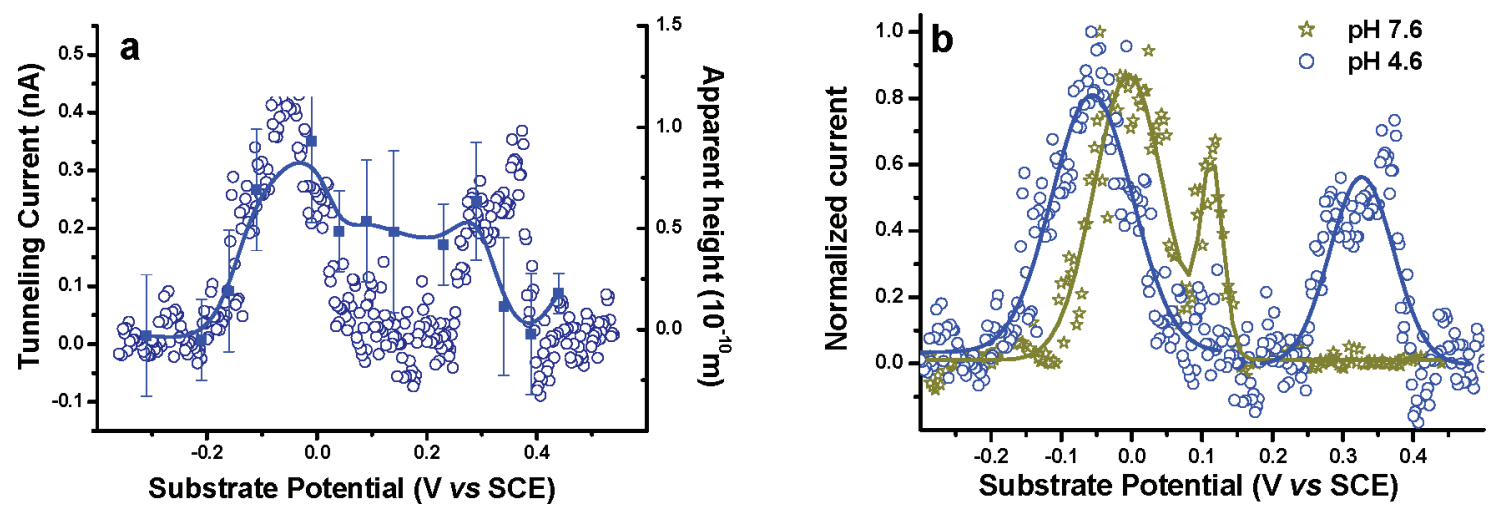

Figure 4. EC-STS analysis of compound 1 on gold. (a) Comparison between EC-STS analysis (tunneling current, circles) and EC-STM imaging (apparent height, squares) performed in $50 \mathrm{mM} \mathrm{NH}_{4} \mathrm{Ac}, \mathrm{pH}$ 4.6. (b) Comparison of EC-STM analysis at pH 4.6 (circles) and 7.6 (stars). (EC-STM: $V_{\text {bias }}=400 \mathrm{mV}, I_{\text {set point }}=1 \mathrm{nA}$; EC-STS: $\left.V_{\text {bias }}=100 \mathrm{mV}, I_{\text {set point }}=0.5 \mathrm{nA}\right)$. The continuous lines in each image are guides for the eye.

by EC-STS for a more basic $\mathrm{pH}$ value. The curve obtained at $\mathrm{pH}$ 7.6 is reported in Figure $4 \mathrm{~b}$ along with a comparison with the data at $\mathrm{pH}$ 4.6. It is evident that the two regions of tunneling enhancement are now nearer to each other. This behavior points to the fact that the two enhancements should be attributed to two different chemical species or redox levels.

If a cyclic voltammetry analysis of the hydroquinone/benzoquinone redox couple is performed in aprotic solvents, the intermediate state involving the exchange of only one electron is stabilized and two peaks appear both in the cathodic and in the anodic scans, each one involving the exchange of one electron. This situation might suggest a possible role of the reduced accessibility of protons to the molecules involved in the electron transfer process as a consequence of the STM tip hindrance. However, the position of the tunneling enhancement peaks observed in the EC-STM and EC-STS is affected by the solution pH, suggesting a role of protons in the overall observed electron transfer mechanism.

In the case of a single redox level between the tip and the substrate in EC-STS configuration, a useful characterization is provided by the analysis of the tunneling current as a function of the substrate potential for different values of the bias voltage. ${ }^{31-35}$ Typically, the relevant comparison is that between the bias voltage and the reorganization energy involved in the redox reaction. In this case, considering the possibility of the involvement of two redox species or two different redox levels, a relevant parameter could also be the comparison of the bias voltage with the separation in energy between the two levels. In Figure 5, the comparison for two bias voltages, $100 \mathrm{vs} 200 \mathrm{mV}$, for the molecule at $\mathrm{pH} 7.6$ (the case with the smaller separation between the peaks) is reported. It is interesting to note that by increasing the bias voltage, a different feature in the tunneling current characteristics appears. The larger bias voltage favors the presence of two plateaus with different values of tunneling current.

3.3. Rationalization of the Differences between Cyclic Voltammetry and EC-STM/STS Investigations: Explanation of the Presence of Two Tunneling Enhancement Regions. Whereas, in cyclic voltammetry, when an electron is transferred from the molecule to the substrate or vice versa, the molecule might change conformation (and rest in the new one), in EC-STM 


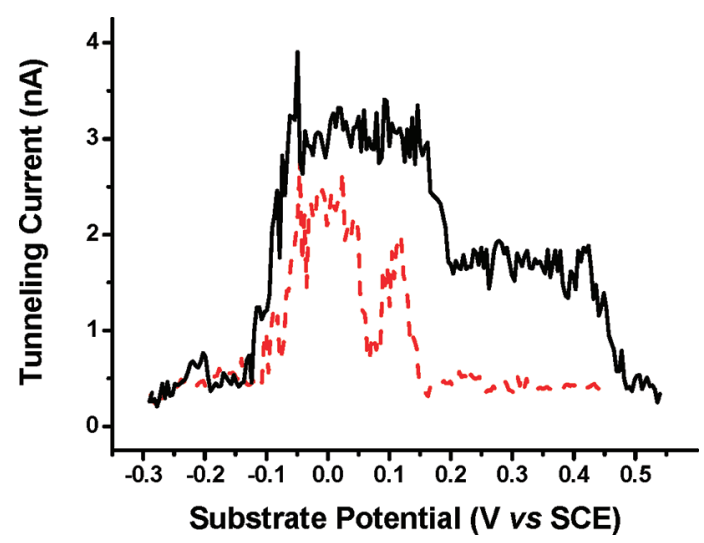

Figure 5. EC-STS analysis of compound 1 on gold at $\mathrm{pH} 7.6$. Comparison between two different bias voltages: 100 (dashed red line) and $200 \mathrm{mV}$ (continuous black line) $\left(I_{\text {set point }}=0.5 \mathrm{nA}\right)$.

experiments, a current flow means a directional steady-state passage of electrons, in the presence of four electrodes, induced by the presence of a bias voltage that might affect in a different way the conformation of the molecule. In the latter case, the current intensity, which is the residing time of electrons on the molecule, can change the extent that the vibrational modes of the molecular structure and the environment are involved in the overall process. ${ }^{6}$ This consideration points to inelastic tunneling contributions to electron transport mechanism and to the peculiarities of in situ measurements with respect to vacuum tunneling, the former contribution being stronger for a long residing time of an electron on a molecule. All of these considerations highlight the differences that might be present in analyzing the electron transport mechanism across a single molecule by the EC-STM setup with respect to a similar case of electrochemical measurements on the same molecule with a three-electrodes setup such as in cyclic voltammetry.

The electron transport mechanism in EC-STM setup involving the energy levels brought about by a redox molecule exchanging one electron at a time has been thoroughly studied in both experimental and theoretical investigations. ${ }^{7-9,11,13,30,36}$ In the case of an asymmetric junction in which the molecule is immobilized on a substrate and an electron tunneling gap is established between substrate and tip, the presence of a redox level induces an increase in the tunneling current at constant bias for a value of substrate potential near the equilibrium potential of the species involved. ${ }^{31}$ This phenomenon is usually referred to as "electrochemical gating”. Many experimental results can be rationalized on the basis of the theoretical framework developed for this particular situation. In this theory, the electron transport mechanism is strictly dependent on vibrational relaxation of the redox species and on fluctuations in the molecular levels due to coupling with the environment. Because of the involvement of environmental properties, it is often referred to as an "in situ" mechanism. It is also possible that in the gap between the tip and the substrate more than one redox level is present. ${ }^{37,38}$ This situation could come into being because of the presence of two redox levels on the same or on two different molecules. Such a situation has recently attracted much theoretical interest due to the possibility of exploiting multielectron systems for molecular electronics devices $^{22,23,27}$. A typical example is represented by the electron transport mechanism in a metallic nanoparticle sandwiched between tip and substrate. ${ }^{34,39}$ In the experimental investigation
Scheme 1. Scheme of the Benzoquinone (Species A) to Hydroquinone (Species C) Reaction through the Semiquinone Species $(\mathrm{B})^{a}$

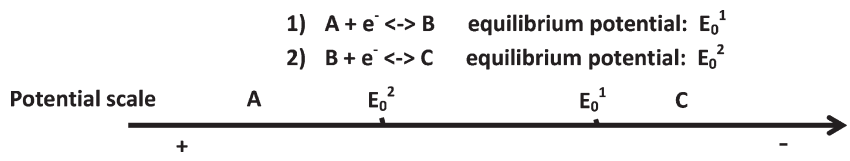

${ }^{a}$ Arrow represents the potential scale pointing to decreasing potential values. The two semi-reactions with the corresponding equilibrium potentials are indicated as reactions 1 and reaction 2 .

presented by Schmickler et al. (ref 37), two redox centers were positioned between two $\mathrm{Hg}$ electrodes. Measuring the current as a function of the potential of one electrode at constant bias, they reported the presence of only one region of current enhancement. Their situation can be theoretically understood in the limit of small coupling of the redox levels with the electrodes, configuring a sort of sequential electron transport, approachable with the kinetic equations methods used for multistep chemical reactions. ${ }^{26}$

The molecules we used in this and in a previous work are characterized by a redox reaction involving two electrons and two protons. In electrochemistry, it is usually assumed that the simultaneous transfer of two electrons is indeed very unlikely. ${ }^{40}$ Usually, multiple electron transfers involve complex reactions that are difficult to depict in a mechanistic fashion. It is also to be stressed that in many cases a particular step in the sequence of all involved transfer events could represent a sort of "rate-limiting step" for the overall reaction. In this case, the electron transfer reaction will be controlled by the difference between applied potential and redox potential of the rate-limiting reaction. This situation exactly applies to our case. Indeed, the hydroquinoneto-benzoquinone reversible redox reaction has been represented by a nine-membered scheme involving the exchange of protons and electrons in sequence. ${ }^{21}$ If protonation reactions are at equilibrium, the nine-membered scheme collapses into a simple reaction with two successive one-electron exchanges. ${ }^{21}$ Depending on the direction of the potential scan, one of the two reactions will be the rate-limiting step for the overall exchange. The possibility of observing the two electron transfer processes as two different peaks (in the anodic or cathodic scans) in a voltammogram depends on the mutual position of the two equilibrium redox potentials. In the case of the hydroquinone/benzoquinone reaction, we can consider Scheme 1, going from positive to negative potentials.

Here species A represents the fully oxidized molecule (benzoquinone), species B (semiquinone) is obtained by adding one electron to species $\mathrm{A}$ (reaction 1 from left to right), and species $\mathrm{C}$ is the fully reduced hydroquinone molecule (obtained by reaction 2 from left to right). The arrow in the scheme represents the potential scale, and it points to decreasing potential values. In this case, it is easy to see that coming from a negative potential, in which the molecule is in state $\mathrm{C}$, the first reaction to occur must be the one controlled by $E_{0}{ }^{2}$ (according to reaction 2 in the scheme), whereas, coming from a positive potential, in which the molecule is in state $A$, the first reaction must be the one controlled by $E_{0}{ }^{1}$ (according to reaction 1 in the Scheme). For a large separation between the two equilibrium potentials, we will see only one wave (anodic or cathodic) in the voltammogram, as in our case. The peak will correspond to the exchange of two electrons, and it will follow an almost Nernstian behavior typical for a 


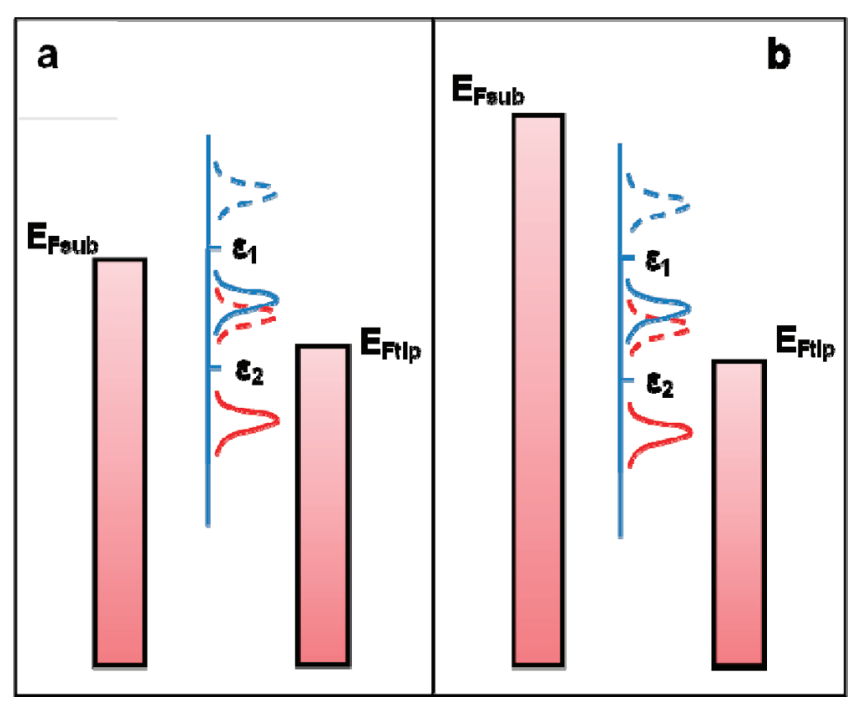

Figure 6. Gerischer model for the redox levels between the energy levels of the tip and the substrate. (a) Low bias voltage with respect to energy levels separation and (b) high bias voltage case.

two electrons - two protons reaction. The particular feature of this reaction, which will be relevant in the discussion of the ECSTM and EC-STS cases, is that one of the two levels (e.g., going from left to right in Scheme 1, level $E_{0}{ }^{1}$ ) does not exist until the potential reaches the value of the second equilibrium potential (e.g., level $E_{0}{ }^{2}$ ). If the positions of the two redox potentials change, then it is possible to observe two separated peaks, as in the case the two equilibrium potentials invert their positions. It is clear that the possibility of observing the two peaks as separated is connected to the interaction between the two involved reactions. If, for example, the electrons are transferred to the same molecular orbital without significant structural rearrangement, then the two electrons will interact according to a strong repulsion, and it will be possible to observe two peaks. If the transfer involves the participation of two redox groups, then the greater the repulsive interaction between the two electrons, the higher the separation between the two peaks. In the case of large molecular or solvation state rearrangements, it is possible that further electrons are attracted, always resulting in the appearance of only one peak. All of the above considerations apply in standard electrochemical investigations such as cyclic voltammetry. At this point, the question relevant to our case is: what happens to the two redox levels of the quinone molecule when they are positioned in the energy (potential) gap defined by the bias voltage in an EC-STM experiment where four electrodes are present? The applied bias voltage and the coupling of the molecule to the two electrodes (the substrate and the tip) will affect the overall reaction mechanism for the exchange of the two electrons. In the Gerischer formalism for electron transfer to solid-state electrodes, we can consider the situations depicted in Figure 6.

In this Scheme, the positions of the two energy levels are affected by the presence of the two electrodes according to the usual mechanism invoked for single electron redox reactions. Different scenarios can apply from now on depending mainly on the strength of the interaction between the molecule and the electrodes and the bias voltage as compared with the separation between the two redox levels and their reorganization energy. ${ }^{23}$ The effect of coupling molecular energy levels to electrodes is that of an increased width of the molecular level distribution and a shift due to a redistribution of the electrons between the molecule and the electrodes. Moreover, the potential at the redox site will be strongly affected by the fraction of the bias voltage and the fraction of the overpotential at the redox site. An analytical treatment of this situation can be found in previous theoretical studies. ${ }^{22,30,39}$ These studies include the cases of a single molecule with multiple redox levels, two molecules each carrying a redox level, and metal nanoparticles with many accessible energy levels. The results of the investigations usually provide the trend of the tunneling current as a function of the substrate overvoltage in a numerical or, upon approximations, analytical form. Here we will try to explain what happens when the substrate potential changes while keeping bias voltage constant. Suppose we are in the situation of Figure 6a with the molecule in state $\mathrm{C}$ according to Scheme 1. Moving the substrate potential toward a more positive value, the first level that can be involved in electron transfer is that related to $\varepsilon_{2}$. At variance with cyclic voltammetry, the exploitable electrode here can be either the tip or the substrate. For a positive tip potential, the level $\varepsilon_{2}$ will be the first to align with the Fermi level of the tip. The transition of one electron from the level $\varepsilon_{2}$ to the tip will cause the involvement in the transfer mechanism also of the level $\varepsilon_{1}$ that could be already in the energy window between the Fermi level of the two electrodes. If this transition takes place when $\varepsilon_{2}$ is still below the Fermi level of the tip, then level $\varepsilon_{2}$ will not contribute to a large extent to the overall tunneling current. In this situation, the main contribution will come from level $\varepsilon_{1}$. In fact, the relaxation of the level $\varepsilon_{1}$ can induce the transfer of many electrons from the substrate to the tip as in the case of the vibrationally coherent twostep electron transfer mechanism in the single redox level case for strong coupling with the electrodes. ${ }^{30}$ If the substrate potential increases further, then the level $\varepsilon_{1}$ will exit from the energy windows defined by the two Fermi levels. When the substrate potential is halfway between the two levels $\varepsilon_{1}$ and $\varepsilon_{2}$, the tunneling current will reach a minimum. Upon further potential increase, the level $\varepsilon_{2}$ will reach the bias voltage window inducing a new maximum in the tunneling current when it is near the Fermi level of the substrate. Each maximum will be caused in this case by the involvement of only one redox level in the electron transport phenomenon. The situation is similar to the case discussed in ref 22 (in that work, the solvent activation mechanism is not considered) even if here the order of the involvement of each level is reversed. The inversion is mainly due to the fact that one of the two levels does not exist until the other reaches its equilibrium potential (see above). The discussed mechanism might induce an asymmetry in the width of the two tunneling current enhancement peaks. The asymmetry will depend on the relative values of the bias voltage, reorganization energy for the two electron transfer processes, and separation between the two redox energy levels.

A different scenario will hold in the case of a large bias voltage with respect to the separation between $\varepsilon_{1}$ and $\varepsilon_{2}$ (Figure $6 \mathrm{~b}$ ) and with respect to the reorganization energy for the single electron transfer. ${ }^{22}$ As soon as level $\varepsilon_{2}$ passes an electron to the tip, level $\varepsilon_{1}$ will be generated and will open a further way for electron transfer. In this case, the tunneling current due to $\varepsilon_{1}$ will reach a short plateau; then, the involvement of level $\varepsilon_{2}$ will increase the tunneling current reaching a further plateau. The plateau is realized when the relaxation of the level in the potential window defined by the bias voltage still keeps the level inside the window. Increasing the positive potential of the substrate, level $\varepsilon_{1}$ will exit from the energy window defined by the bias voltage and the tunneling current will reach the level corresponding to the involvement of 
only level $\varepsilon_{2}$. Then, also level $\varepsilon_{2}$ will go out of the energy window and the tunneling current will be no more enhanced. The abovediscussed mechanism can be used to explain the behavior of the tunneling current reported in Figure 5. The presence of two clear-cut peaks is evident in the case of $100 \mathrm{mV}$ bias voltage. Doubling the bias voltage, we observed the presence of plateaus in the tunneling current, in agreement with the above discussion in the case of large bias voltage. The analysis of the tunneling current also shows the increase in the current intensity between the two plateaus. A similar behavior is predicted in the theoretical investigations reported in ref 22.

\section{CONCLUSIONS}

In the present investigation, we have studied a molecule endowed with two redox levels, which are more closely spaced than in the case of the molecule used in ref 17 . The reduced separation is brought about by the more conductive linker exploited in this work to anchor the benzoquinone/hydroquinone redox group to a $\mathrm{Au}(111)$ substrate. The present case allows us to verify some features predicted by theoretical investigations of a molecule with two redox levels in an EC-STM setup. In fact, the results of Figure 5, clearly show the difference between the application of a small or large bias voltage with respect to the energy level separation, as depicted in Figure 6. These results strongly support the interpretation of the electron transfer mechanism we provided in this work without the need to invoke a coulomb blockade mechanism.

Depending on the value of the bias voltage with respect to the equilibrium potential separation of the two single-electron reactions, the overall process of electron transfer can happen in two separate electron transfer progressions (such as the case presented in Figure 4b), or the two levels can contribute at the same time to the transfer process (such as the case of the continuous line in Figure 5). The particular redox equilibrium potential of the two reactions, essentially related to the configurational molecular and solvent reorganization, makes them impossible to be observed separately in cyclic voltammetry, but they become clearly observable in EC-scanning-tunneling-related methods due to the presence of a second polarizable electrode with which the molecule can exchange electrons. The strong dependence of the studied redox reaction on the environment allows us to regard our system as a molecular switch that can be gated by $\mathrm{pH}$ variations and can sustain multielectron exchanges.

\section{ASSOCIATED CONTENT}

S Supporting Information. Electrochemical characterizations of 4-(2',5'-dihydroxystyryl) benzyl thioacetate SAM on gold including dependence of the peak current and position on the scan rate and dependence on $\mathrm{pH}$. This material is available free of charge via the Internet at http://pubs.acs.org.

\section{AUTHOR INFORMATION}

\section{Corresponding Author}

*E-mail: paolo.facci@unimore.it.

\section{ACKNOWLEDGMENT}

Partial financial support by Italian MIUR FIRB project "Italnanonet" is acknowledged.

\section{REFERENCES}

(1) Aviram, A.; Ratner, M. A. Chem. Phys. Lett. 1974, 29, 277-283.

(2) Nitzan, A.; Ratner, M. A. Science 2003, 300, 1384-1389.

(3) Van der Molen, S. J.; Liljeroth, P. J. Phys.: Condens. Matter. 2010, 22,133001

(4) Weibel, N.; Grunder, S.; Mayor, M. Org. Biomol. Chem. 2007, $5,2343-2353$.

(5) Higgins, S. J.; Nichols, R. J.; Martin, S.; Pilar, C.; Van der Zant, H. S. J.; Richter, M. M.; Low, P. J. Organometallics 2011, 30, 7-12.

(6) Galperin, M.; Ratner, M. A.; Nitzan, A.; Troisi, A. Science 2008, 319, 1056-1060.

(7) Tao, N. J. Phys. Rev. Lett. 1996, 76, 4066-4069.

(8) Schmickler, W. Surf. Sci. 1993, 295, 43-56.

(9) Zhang, J.; Chi, Q.; Albrecht, T.; Kuznetsov, A. M.; Grubb, M.; Hansen, A. G.; Wackerbarth, H.; Welinder, A. C.; Ulstrup, J. Electrochim. Acta 2005, 50, 3143-3159.

(10) Kuznetsov, A. M.; Schmickler, W. Chem. Phys. 2002, 282, 371-377.

(11) Alessandrini, A.; Corni, S.; Facci, P. Phys. Chem. Chem. Phys. 2006, 8, 4383-4397.

(12) Albrect, T.; Guckian, A.; Ulstrup, J.; Vos, J. G. Nano Lett. 2005, 5, 1451-1455.

(13) Li, Z.; Han, B.; Meszaros, G.; Pobelov, I.; Wandlowski, T.; Blaszczyk, A.; Mayor, M. Faraday Discuss. 2006, 131, 121-143.

(14) Facci, P.; Alliata, D.; Cannistraro, S. Ultramicroscopy 2001, 89, 291-298.

(15) Alessandrini, A.; Gerunda, M.; Canters, G. W.; Verbeet, M. P.; Facci, P. Chem. Phys. Lett. 2003, 376, 625-630.

(16) Alessandrini, A.; Salerno, M.; Frabboni, S.; Facci, P. Appl. Phys. Lett. 2005, 86, 133902.

(17) Petrangolini, P.; Alessandrini, A.; Berti, L.; Facci, P. J. Am. Chem. Soc. 2010, 132, 7445-53.

(18) Zhou, X. S.; Liu, L.; Fortgang, P.; Lefevre, A. S.; Serra-Muns, A.; Raouafi, N.; Amatore, C.; Mao, B. W.; Maisonhaute, E.; Schollhorn, B. J. Am. Chem. Soc. 2011, 133, 7509-7516.

(19) Chamber, J. Q. In The Chemistry of the Quinonoid Compounds; Patai, S., Ed.; Wiley: New York, 1974; pp 737-792.

(20) Jacq, J. Electrochim. Acta 1967, 12, 1345-1371.

(21) Laviron, E. J. Electroanal. Chem. 1984, 164, 213-227.

(22) Kuznetsov, A. M.; Ulstrup, J. J. Electroanal. Chem. 2004, 564, 209-222.

(23) Kuznetsov, A. M.; Medvedev, I. G. J. Phys.: Condens. Matter 2008, 20, 374112.

(24) Trammell, S. A.; Seferos, D. S.; Moore, M.; Lowy, D. A.; Bazan, G. C.; Kushmerick, J. G.; Lebedev, N. Langmuir 2007, 23, 942-8.

(25) Bumm, L. A.; Arnold, J. J.; Cygan, M. T.; Dunbar, T. D.; Burgin, T. P.; Jones, L., II; Allara, D. L.; Tour, J. M.; Weiss, P. S. Science 1996, 271, 1705-1707.

(26) Kuznetsov, A. M.; Medvedev, I. G. Electrochem. Commun. 2008, 10, 1191-1194.

(27) Kuznetsov, A. M.; Medvedev, I. G.; Ulstrup, J. J. Chem. Phys. 2009, 131, 164703 .

(28) Tsoi, S.; Griva, I.; Trammell, S. A.; Blum, A. S.; Schnur, J. M.; Lebedev, N. ACS Nano 2008, 2, 1289-1295.

(29) Chi, Q.; Farver, O.; Ulstrup, J. Proc. Natl. Acad. Sci. U.S.A. 2005, 102, 16203-16208.

(30) Friis, E. P.; Kharkats, Y. I.; Kuznetsov, A. M.; Ulstrup, J. J. Phys. Chem. A 1998, 102, 7851-7859.

(31) Pobelov, I. V.; Li, Z.; Wandlowski, T. J. Am. Chem. Soc. 2008, 130, 16045-16054.

(32) Albrecht, T.; Guckian, A.; Kuznetsov, A. M.; Vos, J. G.; Ulstrup, J. J. Am. Chem. Soc. 2006, 128, 17132-8.

(33) Albrecht, T.; Guckian, A.; Ulstrup, J.; Vos, J. G. Nano Lett. 2005, 5, 1451-5.

(34) Li, Z.; Liu, Y.; Mertens, S. F.; Pobelov, I. V.; Wandlowski, T. J. Am. Chem. Soc. 2010, 16, 8187-93. 
(35) Li, C.; Mishchenko, A.; Li, Z.; Pobelov, I.; Wandlowski, T.; Li, X. Q.; Würthner, F.; Bagrets, A.; Evers, F. J. Phys.: Condens. Matter 2008, $20,374122$.

(36) Zhang, J.; Chi, Q.; Kuznetsov, A. M.; Hansen, A. G.; Wackerbath, H.; Christensen, H. E. M.; Andersen, J. E. T.; Ulstrup, J. J. Phys. Chem. B 2002, 106, 1131-1152.

(37) Schmickler, W.; Rampi, M. A.; Tran, E.; Whitesides, G. M. Faraday Discuss. 2004, 125, 171-177; 195-219.

(38) Schmickler, W. Chem. Phys. 2003, 289, 349-357.

(39) Albrecht, T.; Mertens, S. F.; Ulstrup, J. J. Am. Chem. Soc. 2007, 25, 9162-9167.

(40) Bard, A. J.; Faulkner, L. R. Electrochemical Methods: Fundamentals and Applications, 2nd ed.; Wiley: New York, 2001. 